\title{
Genetic polymorphisms of SCN9A are associated with oxaliplatin-induced neuropathy
}

\author{
María Sereno ${ }^{1 *}$, Gerardo Gutiérrez-Gutiérrez², Juan Moreno Rubio', María Apellániz-Ruiz³, Lara Sánchez-Barroso ${ }^{3}$, \\ Enrique Casado1, Sandra Falagan", Miriam López-Gómez", María Merino', César Gómez-Raposo', \\ Nuria Rodriguez-Salas ${ }^{4}$, Francisco Zambrana Tébar ${ }^{1}$ and Cristina Rodríguez-Antona,
}

\begin{abstract}
Background: Oxaliplatin is a chemotherapy agent active against digestive tumors. Peripheral neuropathy is one of the most important dose-limiting toxicity of this drug. It occurs in around $60-80 \%$ of the patients, and $15 \%$ of them develop severe neuropathy. The pathophysiology of oxaliplatin neurotoxicity remains unclear. SCN9A is a gene codifying for a subtype sodium channel (type IX, subunit a) and mutations in this gene are involved in neuropathic perception. In this study we investigated whether SCN9A genetic variants were associated with risk of neurotoxicity in patients diagnosed of cancer on treatment with oxaliplatin.

Methods: Blood samples from 94 patients diagnosed of digestive cancer that had received oxaliplatin in adjuvant or metastatic setting were obtained from three hospitals in Madrid. These patients were classified into two groups: "cases" developed oxaliplatin-induced grade 3-4 neuropathy $(n=48)$, and "controls" $(n=46)$ had no neuropathy or grade 1. The neuropathy was evaluated by an expert neurologist and included a clinical examination and classification according to validated neurological scales: National Cancer Institute Common Toxicity Criteria (NCI-CTC), Oxaliplatin-Specific Neurotoxicity Scale (OSNS) and Total Neuropathy score (TNS). Genotyping was performed for 3 SCN9A missense polymorphisms: rs6746030 (R1150W), rs74401238 (R1110Q) and rs41268673 (P610T), and associations between genotypes and neuropathy were evaluated.
\end{abstract}

Results: We found that SCN9A rs6746030 was associated with protection for severe neuropathy $(\mathrm{OR}=0.39,95 \% \mathrm{Cl}=0$. $16-0.96 ; p=0.041)$. Multivariate analysis adjusting for diabetes provided similar results $(p=0.036)$. No significant differences in neuropathy risk were detected for rs74401238 and rs41268673.

Conclusion: SCN9A rs6746030 was associated with protection for severe oxaliplatin-induced peripheral neuropathy. The validation of this exploratory study is ongoing in an independent series.

Keywords: Oxaliplatin neuropathy, Calcium channel, SCN9A

\section{Background}

Oxaliplatin is a subtype of platinum drugs with significant activity against advanced or metastatic digestive tumors, mainly colorectal cancer (CRC) [1]. Peripheral neuropathy (PN) is a dose-limiting toxicity of oxaliplatin and classically induces two recognized forms of $\mathrm{PN}$ : acute and chronic [2].

\footnotetext{
* Correspondence: mariasereno75@gmail.com

${ }^{1}$ Medical Oncology Department, Infanta Sofía University Hospital, SS de los Reyes, Madrid, Spain

Full list of author information is available at the end of the article
}

Whereas acute PN is not dose-dependent, cumulative doses of oxaliplatin are related to occurrence of chronic peripheral neuropathy [3]. The acute neurotoxicity occurs in a high rate of oxaliplatin-treated patients, and it usually occurs during or within hours of infusion, and it is transient. The majority of the symptoms (including paresthesias and/or dysesthesias in the distal extremities and the perioral region) are typically induced by cold exposition and clinical features include distal sensory neuropathy. Chronic neuropathy is a dose-limiting toxicity defined as a cumulative sensory toxicity. The median 
dose for severe neuropathy is $780-850 \mathrm{mg} / \mathrm{m}^{2}$, and it is seen in $10-15 \%$ of patients [1]. It is a pure sensory, mostly axonal neuropathy with a stocking-and-glove distribution. Symptoms typically are presented between cycles and increase in intensity with cumulative dose. Several factors have been described to increase the risk of severe PN: previous neuropathies, Guillain-Barré syndrome, diabetes mellitus, anemia, hypoalbuminemia, hypomagnesemia and alcohol consumption, as well as have had a recent surgery [4]. However, a large part of the severe neuropathy cases remain unexplained and more studies are needed to discover the underlying factors and to identify patients with a high risk to develop severe PN.

Several markers have been described as potential predictors for acute and chronic oxaliplatin-induced neuropathy (OXLIN) [5-7]. However, none of these have been validated in prospective studies.

The voltage-gated sodium channels are presented in dorsal root ganglion (DRG) and sympathetic ganglion cells, as well as in their small-diameter peripheral axons. They are involved in the initiation and propagation of potentials. They act as gatekeepers of pain at peripheral nociceptors. Nine sodium channel subunits are described (Nav1.1-Nav1.9) [8]. SCN9A gene encodes Nav1.7 isoform. Mutations in SCN9A have been associated with different "channelopathies" with electrical hyperactivity of sensory neurons in dorsal root and a low reactivity of sympathetic ganglia neurons [9]. There are three human pain disorders related to these type of genetic disorders: a bi-allelic loss of function mutations (channelopathy-associated Insensitivity to Pain, CIP), and the opposite situation, an activating mutations with severe episodic pain in Paroxysmal Extreme Pain Disorder (PEPD) and Primary Erythromelalgia (PE) [10]. Moreover; a polymorphism in SCN9A gene (rs6746030) was associated with pain intensity perception in patients with different painful diseases, making DRG neurons more excitable as well as susceptible to aberrant pain perception [11]. Taking into account these evidences, we designed a study to explore the role of SCN9A common genetic variants in OXLIN development in patients diagnosed of digestive tumors that had undergone treatment with oxaliplatin.

\section{Methods}

\section{Patient Selection and OXLIN evaluation}

From 2012 to 2014, one hundred adults with a histologically confirmed diagnosis of digestive tumor (colon, rectum, gastric, pancreatic and biliary duct cancers in different stages) were recruited in three Spanish Hospitals (Infanta Sofía University Hospital, La Paz University Hospital and Infanta Leonor University Hospital). All patients had received an oxaliplatin based regimen (at least $780-800 \mathrm{mg} / \mathrm{m}^{2}$ of oxaliplatin dose) during the last 6 months previous to inclusion. Additional details about the type of tumor and treatment regimens are summarized in Table 1. All cancer patients were over 18 years; life expectancy of $\geq 3$ months; Eastern Cooperative Oncology Group (ECOG) performance status of $\leq 2$; adequate bone marrow, renal and hepatic function; and no previous history of neuropathy. The study was approved by La Paz University Hospital Ethic committee in December 2011 and all patients signed and gave written informed consent.

Inclusion criteria for cases were to have a digestive tumor; receiving oxaliplatin based regimen; cumulative doses $780-850 \mathrm{mg} / \mathrm{m} 2$, and the development of a severe neuropathy (NCI-CTC grade 3-4). Inclusion criteria for controls were also to have a digestive tumor; receiving oxaliplatin based regimen; cumulative dosis 780-850 mg/ $\mathrm{m} 2$ and to have no neuropathy or mild neuropathy (NCICTC grade 0-1). Exclusion criteria for cases and controls was suffering a previous peripheral neuropathy.

The selected 100 patients were divided into two extreme groups: patients with a severe neuropathy and patients without neuropathy after receiving similar regimens and doses of oxaliplatin. This strategy was established to optimize the search of genetic differences between these opposite groups. According to this, fifty patients were defined as "cases", those patients who developed an extreme and severe sensory neuropathy (defined as NCI-CTC grade 3); and fifty patients were defined as "controls", those patients with no neuropathy or grade 1 neuropathy according to clinical scales (NCICTC grade 0-1) after receiving similar doses of oxaliplatin (Table 1).

To perform the clinical evaluation and the neuropathy grading in a homogeneous manner across the different collaborative centers, all patients included in the study underwent a comprehensive neurological examination by the same neurologist as well as neurophysiological studies, performing sural and peroneal conduction studies according to standardized protocols with a Nihon-Kohden electromyography and comparing them to normalized data from the EMG laboratory. The final classification of patients in cases and controls was performed according to the Total Neuropathy Score (TNS) and subscales scores (clinical and reduced, TNSc and TNSr). Cases were considered all those patients with a TNS $>$ or $=15$ and controls those patients with TNS value $<$ or $=6$ (Table 1 ). From the 100 patients recruited, 94 gave blood samples and completed a neurological evaluation and were included in the analysis.

\section{DNA extraction and SNP genotyping}

FlexiGene DNA Kit (Qiagen) was used to isolate DNA from the blood samples of the patients. Final DNA 
Table 1 Demographic and clinical characteristics of the series

\begin{tabular}{|c|c|c|c|}
\hline Variables & $\begin{array}{l}\text { Cases (TNS } \geq 15 ; \\
n=46) \\
N(\%)\end{array}$ & $\begin{array}{l}\text { Controls (TNS } \leq 6 ; \\
n=48) \\
N(\%)\end{array}$ & $P$ value* \\
\hline Sex & & & ns \\
\hline Female & $14(30 \%)$ & $16(33 \%)$ & \\
\hline Male & $32(70 \%)$ & $32(67 \%)$ & \\
\hline Age & & & ns \\
\hline Median (min-max) & $65(41-83)$ & $66(36-82)$ & \\
\hline Diabetes & & & 0.0055 \\
\hline Yes & $11(24 \%)$ & $2(4 \%)$ & \\
\hline No & $35(76 \%)$ & $46(96 \%)$ & \\
\hline Hypothyroidism & & & ns \\
\hline Yes & $0(0 \%)$ & $0(0 \%)$ & \\
\hline No & $46(100 \%)$ & 48 (100\%) & \\
\hline Tumor location & & & ns \\
\hline Colon & $23(50 \%)$ & $30(63 \%)$ & \\
\hline Rectum & $9(20 \%)$ & $7(15 \%)$ & \\
\hline Biliary duct & $2(4 \%)$ & $2(4 \%)$ & \\
\hline Pancreas & $3(6 \%)$ & $4(8 \%)$ & \\
\hline Stomach & $9(20 \%)$ & $5(10 \%)$ & \\
\hline Type of chemotherapy & & & ns \\
\hline XELOX & $35(76 \%)$ & 29 (61\%) & \\
\hline -Adjuvant & 25 & 20 & \\
\hline -Palliative & 10 & 9 & \\
\hline FOLFOX & $6(13 \%)$ & $11(23 \%)$ & \\
\hline -Adjuvant & 3 & 8 & \\
\hline -Palliative & 3 & 3 & \\
\hline TOMOX & $0(0 \%)$ & $2(4 \%)$ & \\
\hline -Adjuvant & 0 & 0 & \\
\hline -Palliative & 0 & 2 & \\
\hline FOLFIRINOX & $1(2 \%)$ & $2(4 \%)$ & \\
\hline -Palliative & 1 & 2 & \\
\hline EOX & $4(9 \%)$ & $4(8 \%)$ & \\
\hline -Perioperative & 4 & 4 & \\
\hline -Palliative & 0 & 0 & \\
\hline Stage AJCC & & & ns \\
\hline$|-| \mid$ & $18(39 \%)$ & $16(33 \%)$ & \\
\hline III-IV & $28(61 \%)$ & $32(67 \%)$ & \\
\hline $\begin{array}{l}\text { Cumulative dose of } \\
\text { oxaliplatin (mg) }\end{array}$ & & & ns \\
\hline Median (min-max) & $1616(880-2045)$ & 1624 (900-2050) & \\
\hline
\end{tabular}

${ }^{*} P$ values $>0.05$ are indicated as non significant (ns) TNS: Total Neuropathy Score $>15$

TNS: Total Neuropathy Score $<6$

analysis was made in 94 patients, 46 cases and 48 controls. Three SNPs in SCN9A (rs41268673, rs6746030 and rs74401238) were selected for genotyping using the
KASPar SNP Genotyping System (LGC Genomics, UK) with $15 \mathrm{ng}$ of genomic DNA. All assays included DNA samples with known genotypes and negative controls. The Sequence Detection System ABI PRISM ${ }^{\circ} 7900 \mathrm{HT}$ (Applied Biosystems) was employed for fluorescence detection and allele assignment.

\section{Statistical analysis}

Clinical variables among cases and controls were compared using Student $t$-test/Mann-Whitney $U$ test (age and cumulative dose of oxaliplatin) or $\mathrm{Chi}^{2}$ test (gender, presence of diabetes mellitus, tumor type, chemotherapy regimen and tumor stage). Association between OXLIN (cases vs controls) and SCN9A polymorphisms was assessed using binary logistic regression analysis. In the multivariate binary logistic regression analysis, diabetes mellitus was included as covariate. We considered an additive genetic model for the SNPs evaluated, and when $P$ value was $<0.1$, other genetic models were explored. Mann-Whitney $U$ test was used to compare TNS grade between the different SCN9A genotypes. The SPSS software package v.19 was used for all statistical analyses. $P$ values less than 0.05 were considered statistically significant.

\section{Results}

The overall demographic and clinical characteristics of the series are represented in Table 1. All patients included received oxaliplatin treatment during at least 6 months (doses $780-850 \mathrm{mg} / \mathrm{m} 2$ ), according to the following protocols: eight courses of XELOX regimen: (Capecitabine) xeloda and oxaliplatin; 12 courses of FOLFOX regimen: 5-fluorouracil, folinic acid (leucovorin) and oxaliplatin; eight courses of TOMOX: raltitrexed and oxaliplatin; six courses of EOX: epirubicin, oxaliplatin and xeloda; or 12 courses of FOLFIRINOX: 5-fluorouacil, folinic acid, irinotecan and oxaliplatin.

The severity of the acute OXLIN was evaluated before every cycle and chronic or persistent toxicity was evaluated up to 3 months after last chemotherapy cycle. According to this, patients were classified in cases and controls according to TNS scale. We established that all those patients with a moderate or severe OXLIN (TNS score $\geq 15$ ) were "cases" (46 patients) and those with inexistent or mild OXLIN (TNS $\leq 6)$ were "controls" (48 patients). The clinical characteristics of these patients were similar, except for diabetes mellitus, which was over-represented in the cases (Table 1).

Among the 94 patients studied, 41 (44\%) patients presented "coasting effect" (that is, a worsening of neuropathy after finishing chemotherapy), 18 (20\%) had dose reductions (around 25-30\%) and 10 (12\%) patients suspended chemotherapy because of the neuropathy. We 
found that all patients with severe acute OXLIN (TNS $\geq 15$ ) also developed a chronic/cumulative disease.

\section{Association between OXLIN and SCN9A genotypes}

With regards to genotyping, $3 S C N 9 A$ missense polymorphisms with a minimum allele frequency $>2 \%$ in the European population (rs6746030 (p.R1150W), rs74401238 (p.R1110Q) and rs41268673 (p.P610T), were genotyped in the 94 patients. We identified $64 \mathrm{C} / \mathrm{C}, 29 \mathrm{C} / \mathrm{T}$ and $1 \mathrm{~T} / \mathrm{T}$ patients for rs6746030 (p.R1150W). For rs41268673 (p.P610T) three individuals were heterozygous and the rest were homozygous wild type, and for rs74401238 (p.R1110Q) five were heterozygous and 89 were homozygous wild type.

We found that rs6746030 variant carriers had significantly lower neuropathy $(\mathrm{OR}=0.39 ; 95 \% \mathrm{CI}=0.16-0.96$; $p=0.041$; dominant model) than wild-type patients. These findings suggest that SCN9A rs6746030 (p.R1150W) variant allele protects against the development of moderate-severe OXLIN (Table 2). Among the different clinical factors analyzed (gender, age, diabetes mellitus, type of tumor, chemotherapy regimen) only diabetes mellitus was significantly associated with moderate-severe OXLIN $(\mathrm{OR}=7.23 ; 95 \% \quad \mathrm{CI}=1.51-34.73 ; p=0.014) \quad$ (Table 3$)$. Multivariate analysis adjusting for diabetes mellitus did not substantially changed the association of SCN9A rs6746030 polymorphism with OXLIN (OR $=0.36 ; 95 \% \mathrm{CI}=0.14$ $0.94 ; p=0.036$ ) (Table 3).

When TNS grade was compared with rs6746030 genotype, in multivariable analysis adjusting by diabetes mellitus, the variant carriers had protection against OXLIN $(\mathrm{OR}=0.89 ; 95 \% \mathrm{CI}=0.81-0.99 ; P=0.044)$.

The other two SCN9A SNPs studied were not associated with OXLIN development in the population included in the study (Table 2). However, the low number of carriers for SCN9A rs74401238 (p.R1110Q) and rs41268673 (p.P610T) indicates a low statistical power in the analysis.

\section{Discussion}

Oxaliplatin peripheral neuropathy affects a large number of patients and can lead to treatment suspension. Most patients recover from the neuropathy in a variable period of time, but long-term nerve damage can also occur, compromising the quality of life of these patients $[12,13]$. Several studies have tried to explain OXLIN mechanism, but, so far, the molecular bases for it remain unknown. In this study we proposed that alterations in $\mathrm{Na}$ channel structure could increase susceptibility for OXLIN development. Previous studies have shown that oxaliplatin causes a functional channelopathy induced by oxalate affecting channels located in cellular membrane [14]. Several preclinical studies with animal models suggested that oxaliplatin modulates the axonal voltage-
Table 2 SCN9A SNPs included in the study with genotype frequencies among cases and controls

\begin{tabular}{|c|c|c|c|c|}
\hline$\overline{S N P}$ & $\begin{array}{l}\text { Total nr. of } \\
\text { patients }\end{array}$ & $\begin{array}{l}\text { Cases TNS } \leq 6 \\
(n=48)\end{array}$ & $\begin{array}{l}\text { Controls TNS } \geq 15 \\
(n=46)\end{array}$ & $P$ value ${ }^{*}$ \\
\hline rs6746030 & & & & 0.041 \\
\hline$C / C$ & 64 & $23(48 \%)$ & 41 (89\%) & \\
\hline$C / T$ & 29 & $24(50 \%)$ & $5(11 \%)$ & \\
\hline $\mathrm{T} / \mathrm{T}$ & 1 & $1(2 \%)$ & $0(0 \%)$ & \\
\hline rs41268673 & & & & 0.589 \\
\hline$C / C$ & 91 & 46 (96\%) & 45 (98\%) & \\
\hline$C / A$ & 3 & $2(4 \%)$ & $1(2 \%)$ & \\
\hline $\mathrm{A} / \mathrm{A}$ & 0 & $0(0 \%)$ & $0(0 \%)$ & \\
\hline rs74401238 & & & & 0.683 \\
\hline $\mathrm{G} / \mathrm{G}$ & 89 & 45 (94\%) & 44 (98\%) & \\
\hline $\mathrm{G} / \mathrm{A}$ & 5 & $3(6 \%)$ & $2(2 \%)$ & \\
\hline $\mathrm{A} / \mathrm{A}$ & 0 & $0(0 \%)$ & $0(0 \%)$ & \\
\hline
\end{tabular}

*Univariate binary logistic regression analysis

gated sodium channels inducing a slow sodium channel kinetics, shifting the voltage dependence to more negative potentials [15]. However, it is not clear what are the mechanisms that justify the development of an acute "sodium channelopathy" in oxaliplatin-treated patients, with inconsistent in vitro studies concerning the role of transient and persistent $\mathrm{Na}+$ conductances [16]. In addition to the "channelopathy hypothesis", other studies have described the "oxidative hypothesis", supported by primary cultures of astrocytes activated in vivo by oxaliplatin treatment. According to the hypothesis of channel disorders induced by oxaliplatin, OXLIN happens when oxaliplatin is accumulated in dorsal root ganglia (DRG) cells. This event, as well as mitochondrial dysfunction with alteration in redox metabolism, is able to induce DRG cells apoptosis $[17,18]$.

Diabetes has been related to a higher risk to develop neuropathy with different anticancer drugs. Kus $\mathrm{T}$ et al. demonstrated in a retrospective analysis that paclitaxelbased therapy in diabetic patients with breast cancer, diabetes duration above 5 years could affect the incidence and severity of PSN without known baseline neuropathy [19]. De la Morena et al. found similar findings in patients with breast cancer treated with paclitaxel every week. They demonstrated that preexisting diabetes

Table 3 Association of rs6746030 with OXLIN under a dominant genetic model

\begin{tabular}{llll}
\hline Variable & OR & $95 \% \mathrm{Cl}$ & $P$ value \\
\hline rs6746030 univariate & 0.39 & $0.16-0.96$ & 0.041 \\
rs6746030 multivariate & 0.36 & $0.14-0.94$ & 0.036 \\
Diabetes mellitus & 7.23 & $1.51-34.73$ & 0.014 \\
\hline
\end{tabular}

${ }^{\mathrm{a}}$ Multivariate binary logistic regression analysis adjusting for diabetes mellitus 
was associated with long-lasting significant neuropathy [20]. As well as with Taxol, several authors have described that diabetic patients are more vulnerable to develop OXLIN. Uwahn AN et al. found that although the presence of diabetes did not appear to affect the severity of OXLIN, patients with diabetes could develop OXLIN with lower doses of oxaliplatin $(P<.05)[21]$.

However, no molecular studies have identified markers able to predict OXLIN development. Argyriou A et al. found polymorphisms in different voltage-gated sodium channels with a potential role in predicting severe acute and chronic OXLIN. They found that the experimental model of the skeletal muscle sodium channel SCN $4 A$ rs2302237 and the tetrodotoxin-resistant SCN10A rs1263292 polymorphisms could be related to the development of acute OXLIN [22, 23].

SCN9A encodes Nav1.7 isoform and it plays an important role in defining of threshold for excitation of nociceptors and can control neurotransmitter release at the terminals of these receptors. Homozygous loss of function mutations in $\mathrm{Na}_{\mathrm{v}} 1.7$ has been related to a congenital inability to suffer pain and anosmia in men. In addition, heterozygous gain of function mutations have been described in different clinical pain syndromes of inherited erythromelalgia (IEM: pain and erythema exacerbated by warming), paroxysmal extreme pain disorder (PEPD: proximal pain and autonomic features in ocular/ mandibular and sacral regions), and small fiber neuropathy (SFN: degeneration of small diameter sensory and autonomic axons presenting with a severe burning pain) [24]. As we already have mentioned, different mutations in SCN9A gene could cause chronic pain and pain insensitivity syndromes [25]. For example, Peddareddygari $L R$ et al. demonstrated that homozygous p.G2755T mutation in exon 15 of this gene is associated with congenital insensitivity to pain (CIP) $[26,27]$. Other authors have related some mutations like p.L1612P with a Cold Sensitive Paroxysmal Extreme Pain Disorder [28]. According to these data and the role of this gene in pain and cold perception, we decided to analyzed SCN9A missense SNPs (rs6746030 (p.R1150W), rs74401238 (p.R1110Q) and rs41268673 (p.P610T)) as a potential cause of genetic susceptibility for a cold-induced neuropathy like OXLIN. We found that SCN9A rs6746030 variant allele protected against the development of moderate-severe OXLIN in univariate and multivariate analysis ( $p=0.041$ and $p=0.036$, respectively).

A validation in a prospective series of patients receiving oxaliplatin is needed to confirm this association. We could not obtain any conclusions about the other two SNPs because of low frequency in European population.

In addition, we analyzed the correlation between chronic and acute OXLIN. We observed that all patients considered "cases", with severe acute OXLIN, also had a moderate-severe chronic/cumulative neuropathy. Argyriou et al. also demonstrated this correlation in a cohort of patients with OXLIN. A possible explanation for this could be the cellular stress affecting the sensory cells as a result of the prolonged activation of SCNAs, inducing a decrease of neuron metabolism and axoplasmic function in the DRG cells [18]. Preclinical data with sural nerve cultures exposed to oxaliplatin showed alterations in sodium channel kinetics and prolonged opening of sodium channels resulting in an increase in sodium currents [29].

\section{Conclusion}

In conclusion, OXLIN is a multifactorial disease. Diabetes mellitus and previous neuropathy increase the risk for developing OXLIN; however, so far, no genetic predisposition markers have been defined. In this study, we found that SCN9A rs6746030 (p.R1150W) variant allele is associated with a protective effect against developing moderate-severe OXLIN. Prospective evaluation of these findings is needed to confirm the role of this $S C N 9 \mathrm{~A}$ polymorphism in OXLIN development.

\section{Abbreviations \\ CIP: Channelopathy associated insensitivity to pain; CIP: Congenital Insentitivity to pain; CRC: Colorectal cancer; DRG: Dorsal root ganglia; ECOG: Eastern Cooperative Oncology Group; IEM: Inherited erythromelalgia; NCl-CTC: National Cancer Institute-Common Toxicity Criteria; \\ OSNS: Oxaliplatin Specific Neuropathy Scale; OXLIN: Oxaliplatin Induced neuropathy; PD: Paroxysmal Extreme Pain Disorders; PE: Primary Erithromelalgia; PEDP: Paroxysmal extreme pain disorder; PN: Peripheral neuropathy; SFN: Small fiber neuropathy; TNS: Total Neuropathy Scale}

\section{Acknowledgements}

For all the patients who accepted to participate in this investigation.

\section{Funding}

This work was supported by projects from the "Spanish Ministry of Economy and Competiveness" (grant number SAF2015-64850-R) and from Carlos III Health Institute project: PI12/02824.

\section{Availability of data and materials}

The datasets used and/or analysed during the current study available from the corresponding author and Rodriguez Antona C.

\section{Authors' contributions}

MS recruited patients for the study and drafted the manuscript. GG carried out the neurological examination. JM participated in the molecular analysis. MA-R and LS-B participated in molecular and statistical analysis. SF, MM, EC, ML, NR-S, CG, FZ participated in patients' recruitment, its design and coordination and helped to draft the manuscript. CR-A coordinated the study and helped with the draft of the manuscript. All authors read and approved the final manuscript.

\section{Authors' Information}

María Sereno MD, PhD, Oncologist, Medical Oncology Department, Infanta Sofía University Hospital, Madrid

Gerardo Gutiérrez MD, Neurologist, Neuromuscular Disease Department, Infanta Sofía University Hospital, Madrid

Juan Moreno; Molecular Biologist, Precision Oncology Laboratory, Infanta Sofía University Hospital, Madrid

María Apellániz Ruiz and Lara Sánchez-Barroso, Nurse research and Biologist in Oncology Institute of Cancer (CNIO) research in Madrid

Sandra Falagan, Maria Merino, Enrique Casado, Miriam López, Nuria RodriguezSalas, César Gómez, Francisco Zambrana; MD, Oncologists, Medical Oncology Department, Infanta Sofía University Hospital, Madrid 
Cristina Rodríguez-Antona; Biologist in Oncology Institute of Cancer (CNIO) research in Madrid

\section{Competing interests}

María Sereno has not financial competing interests to declare. Gerardo Gutiérrez has not financial competing interests to declare. Juan Moreno; has not financial competing interests to declare. María Apellániz Ruiz and Lara Sánchez-Barroso has not financial competing interests to declare.

Sandra Falagan, Maria Merino, Enrique Casado, Miriam López, Nuria RodriquezSalas, César Gómez, Francisco Zambrana; has not financial competing interests to declare.

Cristina Rodríguez-Antona; has not financial competing interests to declare.

\section{Consent for publication}

All the patients included accepted in then initial written informed consent the possibility that the results of this work could be published.

\section{Ethics approval and consent to participate}

The study was approved by La Paz University Hospital Ethic committee in December 2011 and all patients gave written informed consent.

\section{Author details}

${ }^{1}$ Medical Oncology Department, Infanta Sofía University Hospital, SS de los Reyes, Madrid, Spain. ${ }^{2}$ Neurology Department, Infanta Sofía University Hospital, SS de los Reyes, Madrid, Spain. ${ }^{3}$ Hereditary Endocrine Cancer Group, Spanish National Cancer Research Centre (CNIO), Madrid, Spain. ${ }^{4}$ Medical Oncologist, Medical Oncology Department, La Paz University Hospital, Madrid, Spain. ${ }^{5}$ Hereditary Endocrine Cancer Group, Human Cancer Genetics Programme, Spanish National Cancer Center (CNIO), Madrid, Spain. ${ }^{6}$ SCIII Center for Biomedical Research on Rare Diseases (CIBERER), Madrid, Spain.

\section{Received: 20 August 2016 Accepted: 13 December 2016}

\section{Published online: 19 January 2017}

\section{References}

1. Sereno M, Gutiérrez-Gutiérrez G, Gómez-Raposo C, López-Gómez M, Merino- Salvador M, et al. Oxaliplatin induced-neuropathy in digestive tumors. Crit Rev Oncol Hematol. 2014;89:166-78.

2. Loprinzi CL, Qin R, Dakhil SR, Fehrenbacher L, Flynn KA, Atherton P, et al. Phase III randomized, placebo-controlled, double-blind study of intravenous calcium and magnesium to prevent oxaliplatin-induced sensory neurotoxicity (N08CB/Alliance). J Clin Oncol. 2014;32:997-1005.

3. Argyriou AA, Velasco R, Briani C, Cavaletti G, Bruna J, Alberti $P$, et al. Peripheral neurotoxicity of oxaliplatin in combination with 5-fluorouracil (FOLFOX) or capecitabine (XELOX): a prospective evaluation of 150 colorectal cancer patients. Ann Oncol. 2012;23:3116-22.

4. Nakayama G, Kodera Y, Yokoyama H, Okuda N, Watanabe T, Tanaka C, et al. Modified FOLFOX6 with oxaliplatin stop-and-go strategy and oral S-1 maintenance therapy in advanced colorectal cancer: CCOG-0704 study. Int J Clin Oncol. 2011;16:506-11.

5. Won HH, Lee J, Park JO, Park YS, Lim HY, Kang WK, et al. Polymorphic markers associated with severe oxaliplatin-induced, chronic peripheral neuropathy in colon cancer patients. Cancer. 2012;118:2828-36.

6. Inada M, Sato M, Morita S, Kitagawa K, Kawada K, Mitsuma A, et al. Associations between oxaliplatin-induced peripheral neuropathy and polymorphisms of the ERCC1 and GSTP1 genes. Int J Clin Pharmacol Ther. 2010;48:729-34.

7. Kumamoto K, Ishibashi K, Okada N, Tajima Y, Kuwabara K, Kumagai Y, et al. Polymorphisms of GSTP1, ERCC2 and TS-3'UTR are associated with the clinical outcome of mFOLFOX6 in colorectal cancer patients. Oncol Lett. 2013;6:648-54

8. Houlden $\mathrm{H}$. Extending the clinical spectrum of pain channelopathies. Brain 2012;135:313-6.

9. Vargas-Alarcon G, Alvarez-Leon E, Fragoso JM, Vargas A, Martinez A, Vallejo $M$, et al. A SCN9A gene-encoded dorsal root ganglia sodium channel polymorphism associated with severe fibromyalgia. BMC Musculoskelet Disord. 2012;13:23.

10. Cox JJ, Sheynin J, Shorer Z, Reimann F, Nicholas AK, Zubovic L, et al. Congenital insensitivity to pain: novel SCN9A missense and in-frame deletion mutations. Hum Mutat. 2010;31:E1670-86.
11. Reimann F, Cox JJ, Belfer I, Diatchenko L, Zaykin DV, McHale DP, et al. Pain perception is altered by a nucleotide polymorphism in SCN9A. Proc Natl Acad Sci U S A. 2010;107:5148-53.

12. Cornblath DR, Chaudhry V, Carter K, Lee D, Seysedadr M, Miernicki M, John T, et al. Total neuropathy score: validation and reliability study. Neurology. 1999:53:1660-4.

13. Padman S, Lee J, Kumar R, Slee M, Hakendorf P, Richards A, et al. Late effects of oxaliplatin-induced peripheral neuropathy (LEON) - cross-sectional cohort study of patients with colorectal cancer surviving at least 2 years. Support Care Cancer. 2015;23:861-9.

14. Park SB, Lin CS, Krishnan AV, Goldstein D, Friedlander ML, Kiernan MC. Dose effects of oxaliplatin on persistent and transient $\mathrm{Na}+$ conductances and the development of neurotoxicity. PLoS One. 2011;6:e18469.

15. Adelsberger H, Quasthoff S, Grosskreutz J, Lepier A, Eckel F. The chemotherapeutic oxaliplatin alters voltage-gated $\mathrm{Na}(+)$ channel kinetics on rat sensory neurons. Eur J Pharmacol. 2000;406:25-32.

16. Wu S-N, Chen B-S, Wu Y-H, Peng H, Chen L-T. The mechanism of the actions of oxaliplatin on ion currents and action potentials in differentiated Ng108-15 neuronal cells. Neurotoxicology. 2009:30:677-85.

17. Di Cesare Mannelli L, Zanardelli M, Failli P, Ghelardini C. Oxaliplatin-induced oxidative stress in nervous system-derived cellular models: Could it correlate with in vivo neuropathy? Free Radic Biol Med. 2013;61:143-50.

18. Argyriou AA, Bruna J, Marmiroli P, Cavaletti G. Chemotherapy-induced peripheral neurotoxicity (CIPN): an update. Crit Rev Oncol Hematol. 2012;82:51-77.

19. Kus T, Aktas G, Kalender ME, Sevinc A, Kul S, Suner A, et al. Taxane-induced peripheral sensorial neuropathy in cancer patients is associated with duration of diabetes mellitus: a single-center retrospective study. Support Care Cancer. 2016;24:1175-9.

20. de la Morena Barrio P, Conesa MÁ, González-Billalabeitia E, Urrego E, GarcíaGarre E, García-Martínez E, et al. Delayed recovery and increased severity of Paclitaxel-induced peripheral neuropathy in patients with diabetes. J Natl Compr Cancer Netw. 2015;13:417-23.

21. Uwah AN, Ackler J, Leighton Jr JC, Pomerantz S, Tester W. The effect of diabetes on oxaliplatin-induced peripheral neuropathy. Clin Colorectal Cancer. 2012:11:275-9.

22. Argyriou AA, Cavaletti G, Antonacopoulou A, Genazzani AA, Briani C, Bruna J, et al. Voltage-gated sodium channel polymorphisms play a pivotal role in the development of oxaliplatin-induced peripheral neurotoxicity: results from a prospective multicenter study. Cancer. 2013:119:3570-7.

23. Huang J, Han C, Estacion M, Vasylyev D, Hoeijmakers JG, Gerrits MM, PROPANE Study Group, et al. Gain-of-function mutations in sodium channel $\mathrm{Na}(\mathrm{V}) 1.9$ in painful neuropathy. Brain. 2014;137:1627-42

24. Harrer JU, Uçeyler N, Doppler K, Fischer TZ, Dib-Hajj SD, Waxman SG, et al. Neuropathic pain in two-generation twins carrying the sodium channel Nav1.7 functional variant R1150W. Pain. 2014;155:2199-203.

25. Holliday KL, Thomson W, Neogi T, Felson DT, Wang K, Wu FC, et al. The non-synonymous SNP, R1150W, in SCN9A is not associated with chronic widespread pain susceptibility. Mol Pain. 2012;8:72.

26. Duan G, Xiang G, Zhang X, Yuan R, Zhan H, Qi D. A single-nucleotide polymorphism in SCN9A may decrease postoperative pain sensitivity in the general population. Anesthesiology. 2013;118:436-42.

27. Peddareddygari LR, Oberoi K, Grewal RP. Congenital insensitivity to pain: a case report and review of the literature. Case Rep Neurol Med. 2014:2014:141953.

28. Suter MR, Bhuiyan ZA, Laedermann CJ, Kuntzer T, Schaller M, Stauffacher MW, et al. p.L1612P, a novel voltage-gated sodium channel Nav1.7 mutation inducing a cold sensitive paroxysmal extreme pain disorder. Anesthesiology. 2015;122:414-23.

29. Sittl R, Lampert A, Huth T, Schuy ET, Link AS, Fleckenstein J, et al. Anticancer drug oxaliplatin induces acute cooling-aggravated neuropathy via sodium channel subtype $\mathrm{Na}(\mathrm{V}) 1.6$-resurgent and persistent current. Proc Natl Acad Sci USA. 2012:109:6704-9. 